\title{
Correction to: Biobased Nanotechnology for Green Applications
}

\author{
Hemen Sarma, Sanket J. Joshi, Ram Prasad, and Josef Jampilek
}

\section{Correction to:}

\section{H. Sarma et al. (eds.), Biobased Nanotechnology for Green Applications, Nanotechnology in the Life Sciences, https://doi.org/10.1007/978-3-030-61985-5}

Chapter 7 in: H. Sarma et al. (eds.), Biobased Nanotechnology for Green Applications, Nanotechnology in the Life Sciences, https://doi.org/10.1007/978-3-030-61985-5_7

Chapter 9 in: H. Sarma et al. (eds.), Biobased Nanotechnology for Green Applications, Nanotechnology in the Life Sciences, https://doi.org/10.1007/978-3-030-61985-5_9

Owing to an error on the part of the editor and corresponding chapter author, the author Hossein Motamedi has been removed from the chapter 7 Biosynthesis of Nanoparticles from Bacteria and Thallophytes: Recent Advances and chapter 9 Microbial Nanotechnology in Life Sciences: An Opportunity for Green Applications. The author list has now been updated by removing Hossein Motamedi in the chapter opening pages, table of contents, and wherever applicable throughout the book.

The updated version of these chapters can be found at https://doi.org/10.1007/978-3-030-61985-5_7 https://doi.org/10.1007/978-3-030-61985-5_9 https://doi.org/10.1007/978-3-030-61985-5 